\section{Plasma Mevalonate as a Measure of Cholesterol Synthesis in Man}

\author{
Thomas S. Parker, Donald J. McNamara, \\ Clinton D. Brown, Rachael Kolb, and E. H. Ahrens, Jr. \\ The Rockefeller University Hospital, New York, NY 10021
}

Alfred W. Alberts, Jonathan Tobert, and Julie Chen Merck Sharp \& Dohme Research Laboratories, Rahway, NJ 07065

Paul J. De Schepper

University of Leuven, B-3000 Leuven, Belgium
A (MVA) concentrations in plasma or 24-h urine samples is shown to be useful in studies of the regulation of 3-hydroxy-3-methylglutaryl coenzyme A (HMG-CoA) reductase and cholesterol synthesis. Plasma MVA concentrations, measured either at 7-9 a.m. after an overnight fast, or throughout the 24-h cycle, were compared with cholesterol synthesis rates that were measured by the sterol balance method: plasma MVA concentrations were directly related to the rate of whole body cholesterol synthesis $(r=0.972 ; p<0.001 ; n=18)$ over a tenfold range of cholesterol synthesis rates. Moreover, hourly examination of MVA concentrations throughout the day demonstrated that interventions such as fasting or cholesterol feeding cause suppression of the postmidnight diurnal rise in plasma MVA concentrations, with little change in the base-line of the rhythm. Thus, the daily rise and fall of plasma MVA appears to reflect changes in tissues and organs, such as the liver and intestine, that are known to be most sensitive to regulation by fasting or by dietary cholesterol.

The hypothesis that short-term regulation of HMGCoA reductase in tissues is quickly reflected by corresponding variations in plasma MVA was tested by using a specific inhibitor of HMG-CoA reductase, mevinolin, to block MVA synthesis. Mevinolin caused a dosedependent lowering of plasma MVA after a single dose; and in patients who received the drug twice a day for 4 wk, it decreased 24-h urinary MVA output. Significant

Received for publication 28 September 1983 and in revised form 14 May 1984.

J. Clin. Invest.

(C) The American Society for Clinical Investigation, Inc. 0021-9738/84/09/0795/10 $\$ 1.00$

Volume 74, September 1984, 795-804 lowering of plasma cholesterol was achieved through administration of mevinolin at doses that only moderately limit MVA production.

\section{Introduction}

The biological mechanisms that regulate cholesterol metabolism and maintain cholesterol homeostasis often oppose dietary, pharmacological, or surgical interventions that are aimed at lowering plasma cholesterol levels $(1,2)$. Because most of the cholesterol in man arises by de novo synthesis (3), interest has focused on understanding the mechanisms of regulation of the rate-limiting enzyme of this metabolic pathway, 3-hydroxy-3methylglutaryl coenzyme A (HMG-CoA) ${ }^{1}$ reductase (mevalonate:NADP oxidoreductase [acylating CoA], EC 1.1.1.34), and on the development of inhibitors as pharmacological agents for limiting cholesterol production and lowering plasma cholesterol levels in hypercholesterolemic patients.

HMG-CoA reductase activity is usually measured in vitro by quantitating the rate of formation of mevalonic acid (MVA) from radioactive HMG-CoA. However, MVA can be detected in the blood plasma $(4,5)$ in concentrations that shift in parallel with changes in liver HMG-CoA reductase in the rat (5), and with changes in whole body cholesterol synthesis in man (6). A diurnal rhythm of plasma MVA concentrations has been described in human subjects and shown to be suppressed by dietary cholesterol or fasting $(6,7)$. Turnover of labeled MVA is biphasic and rapid $\left(t^{1 / 2}<1 \mathrm{~h}\right)$ in both the rat and man $(5,7)$ and clearance, primarily by the kidney $(8,9)$, but also by the liver and intestine (10), does not appear to be saturated at physiological concentrations. These observations have led us to the conclusion that plasma MVA concentrations are determined by the balance between MVA input, which is driven by intracellular MVA synthesis in response to factors that regulate HMG-CoA reductase activity, and output, which is mediated by concentration-dependent mechanisms.

1. Abbreviations used in this paper: HMG-CoA, 3-hydroxy-3-methylglutaryl coenzyme A; MVA, mevalonic acid. 
We have proposed that the presence in human plasma of MVA, an obligate, rate-limiting precursor of cholesterol, in amounts that are apparently related to the rate of cholesterol synthesis, offers a noninvasive, nonisotopic method for studying the regulation of cholesterol synthesis in man (6). In the studies presented here, this hypothesis is tested by comparing plasma MVA concentrations and cholesterol synthesis rates under a variety of conditions; the data demonstrate that the two are directly related over a tenfold range of synthesis rates. Through analysis of isotopic MVA kinetics and consideration of the effect of cholesterol feeding on the diurnal MVA rhythm, we explore the possible mechanisms that link plasma MVA and cholesterol synthesis; and by characterizing the effects on MVA concentrations of mevinolin, a specific inhibitor of HMG-CoA reductase $(11,12)$, we demonstrate the usefulness of MVA measurements in clinical studies of cholesterol synthesis.

\section{Methods}

Experimental design. Informed consent for designs I, II, and III below were obtained from all in-patients after appropriate review and approval of study protocols by The Rockefeller University Hospital Institutional Review Board. Clinical data and lipid phenotypes for these patients are given in Table I.

I. Correlation between cholesterol synthesis and plasma MVA concentrations. Sterol balance studies were carried out on 11 patients (Table I) who were admitted to the metabolic ward at The Rockefeller University Hospital. Except where specifically noted, all patients were maintained solely on liquid formula feeding that was divided into five equal portions and fed to them at 8 a.m. and thereafter at $\sim 3-\mathrm{h}$ intervals. Caloric intakes were adjusted to maintain body weights that were constant within $\pm 1.5 \mathrm{~kg}$ over study periods of 3-5 wk. The preparation of liquid formula diets and supplementation with vitamins and minerals has been described (13). The cholesterol content of the formulas was adjusted by dissolving cholesterol (Sigma Chemical Co., St. Louis, MO) after recrystallization from ethanol in the oil phase, during formula preparation.

An adaptation period of at least $2 \mathrm{wk}$ allowed patients to achieve a metabolic steady state, as indicated by constancy of body weight, plasma cholesterol concentration, and daily output of fecal sterols, and a stable clinical course. Stools for sterol balance measurements were collected and analyzed as described elsewhere $(14,15)$, with chromic oxide (16) and sitosterol (17) as internal standards. Blood samples for routine MVA determination were obtained by venipuncture between 7 and 9 a.m. after an overnight fast.

II. Diurnal variation of plasma MVA levels. Except where specifically noted, diurnal MVA rhythms were examined after an adaptation period of three or more weeks on the metabolic ward. A heparin lock that had been placed in the antecubital vein allowed patients to move about or sleep while blood samples were drawn throughout the $24 \mathrm{~h}(6)$.

III. Plasma MVA kinetics. The rate of plasma MVA turnover was measured by the constant isotope infusion method using $3 \mathrm{R}\left[5-{ }^{3} \mathrm{H}\right] \mathrm{MVA}$ $(2,000 \mathrm{Ci} / \mathrm{mol}, \mathrm{sp}$ act). A priming dose $(0.61 \mu \mathrm{Ci} / \mathrm{kg})$ and a constant infusion $(1.27 \mu \mathrm{Ci} / \mathrm{min})$ of $3 \mathrm{R}\left[5-{ }^{-} \mathrm{H}\right] \mathrm{MVA}$ were administered through an antecubital vein while samples were taken from the contralateral vein. Samples were analyzed for MVA mass and $3 R\left[5-{ }^{3} \mathrm{H}\right] \mathrm{MVA}$ (see below) in order to calculate plasma MVA specific activity.

IV. Effects of mevinolin. MVA and mevinolin concentrations were measured in samples of plasma that were obtained from eight volunteers who participated in a preliminary double-blind, placebo-controlled, single-dose study of mevinolin that was carried out by one of us (Dr. De Schepper). These patients were healthy males of mean age 22 (2125) yr and mean weight $73.3(63-91) \mathrm{kg}$.

Daily urinary MVA output was measured in samples that were obtained from 37 patients who were administered mevinolin twice a day for $4 \mathrm{wk}$ in a multicenter, double-blind, placebo-controlled, doseranging study of mevinolin (12). These patients were healthy male volunteers of mean age $28.6(18-35)$ yr, who had plasma cholesterol levels that were between 150 and $300 \mathrm{mg} / \mathrm{dl}$.

Certain data obtained in these drug studies have been reported previously (12); informed consents were obtained according to the practices of the participating institutions.

\section{Analytical procedures}

Preparation of $3 R\left[5-{ }^{3} \mathrm{H}\right] M V A$. $3 \mathrm{RS}\left[5-{ }^{3} \mathrm{H}\right] \mathrm{MVA}$ lactone $(2,000 \mathrm{Ci} / \mathrm{mol}$, sp act) was purchased from New England Nuclear, Boston, MA, hydrolyzed, and converted to 5-phospho-3R $\left[5-{ }^{3} \mathrm{H}\right] \mathrm{MVA}$ by incubation with partially purified pig liver mevalonate kinase (18). The 5-phospho$3 \mathrm{R}\left[5-{ }^{3} \mathrm{H}\right] \mathrm{MVA}$ was purified by liquid column chromatography on Dowex $1 \times 8$ (Bio-Rad Laboratories, Inc., Richmond, CA) as previously described (5), converted to $3 \mathrm{R}\left[5-{ }^{3} \mathrm{H}\right] \mathrm{MVA}$ by incubation with alkaline phosphatase, then made $90 \%$ in ethanol to precipitate buffer salts and protein before dilution into water $(1: 10)$ for rechromatography, as above. The product was lactonized $(0.1 \mathrm{~N} \mathrm{HCl})$ and further purified by TLC on Silica gel-G (Eastman 13179, Eastman Kodak Co., Rochester, NY) with acetone-benzene $(1: 1 \mathrm{v} / \mathrm{v})$ as the solvent. Pure 3R[5${ }^{3} \mathrm{H}$ ]MVA lactone was eluted from silica gel (recovery $75 \%$ ) and stored in ethanol at $4^{\circ} \mathrm{C}$ until use. This procedure routinely gave sterile and pyrogen-free preparations of $3 \mathrm{R}\left[5-{ }^{3} \mathrm{H}\right] \mathrm{MVA}$. For clinical studies, $3 \mathrm{R}$ [5$\left.{ }^{3} \mathrm{H}\right] \mathrm{MVA}$ was dissolved in sterile saline and administered intravenously.

Determination of MVA mass and specific activity. Plasma MVA was measured by the radioenzymatic method of Popják et al. (5), which was modified as previously described (6). Urine samples were prepared for MVA determination by centrifugation $(2,000 \times g \times 20$ $\mathrm{min}$ ) to remove solids, then sterilized by filtration (Millipore: $0.45 \mu \mathrm{m}$ ) and assayed as above.

$3 R\left[5-{ }^{3} \mathrm{H}\right] \mathrm{MVA}$ was quantitated by incubation of protein-free plasma ultrafiltrates (Amicon CF25 membranes; Amicon Corp., Bedford, MA) with pig liver mevalonate kinase and ATP, followed by chromatography of the products on Dowex $1 \times 8$ as described above except that 3RS[5${ }^{14}$ C]MVA (Research Products International Co., Mount Prospect, IL; $39.5 \mathrm{Ci} / \mathrm{mol}$, sp act) was included in the incubation as an internal standard to correct for efficiency of conversion to phosphomevalonate and recovery (assuming 50\% $3 \mathrm{R}\left[5-{ }^{14} \mathrm{C}\right] \mathrm{MVA}$ ). Column fractions that contained ${ }^{3} \mathrm{H}$ - and ${ }^{14} \mathrm{C}$-phosphomevalonate were dispersed in scintillant (Hydrofluor, New England Nuclear, Boston, MA) and counted in a Packard (3990) tricarb liquid scintillation spectrometer (Packard Instrument Co., Downers Grove, IL) that was calibrated to correct for efficiency and crossover using an external standard. By this method, $70-80 \%$ of the total tritium in protein-free plasma ultrafiltrates was present as $3 \mathrm{R}\left[5-{ }^{3} \mathrm{H}\right] \mathrm{MVA}$ and most of the remainder was ${ }^{3} \mathrm{H}_{2} \mathrm{O}$ (as isolated by lyophilization): ${ }^{3} \mathrm{H}$-labeled lipids that were bound to proteins or carried on plasma lipoproteins were excluded from the plasma ultrafiltrates and did not interfere with determination of $3 \mathrm{R}\left[5-{ }^{3} \mathrm{H}\right] \mathrm{MVA}$. 
Mevinolin assay. Mevinolin was quantitated by comparing the degree of inhibition that was produced by mevinolin-containing samples in a standard HMG-CoA reductase assay (19). Plasma or urine (0.1 $\mathrm{ml}$ ) were prepared for assay by adding $0.1 \mathrm{ml}$ of methanol followed by centrifugation to remove precipitates. A standard curve was generated by adding known quantities of the hydroxy acid form of mevinolin to aliquots of normal human plasma. Results are expressed as equivalents of biologically active mevinolinic acid (nanomole per liter).

Statistical analysis. Program P7R of the Biomedical Computer Program Series (BMDP), which was developed at the UCLA Health Sciences Computing Facility (1976 revision), Los Angeles, CA, was used for regression analysis. In applying the linear regression method to analyze plasma MVA concentration and cholesterol synthesis rate data, we tested the hypothesis that these variables would be correlated over a broad range of synthesis rates and under a wide variety of conditions. Thus, data collected from individuals who represented groups that had various cholesterol synthesis rates (i.e., normal weight vs. obese) were pooled with data that was collected from individuals who were studied before and during interventions that were known to alter synthesis rates (i.e. cholestyramine, portacaval anastomosis (PCA), diet), and all data pairs were assumed to have the same degree of statistical independence and were given equal weight.

Regression analysis was also used to test for a relationship between mevinolin dose and 24-h urinary MVA output. In this analysis, a log transformation was used to obtain values of $1,2,3$, and 4 on the independent variable (dose of mevinolin $0,6.25,12.5,25$, and $50 \mathrm{mg}$ b.i.d.). In cases where the results of linear regression analysis were strongly influenced by a small number of data pairs that were clustered far from the sample mean (Tables I and II), a nonparametric test of correlation, Spearman's rank correlation coefficient $\left(r_{s}\right)$, was reported together with the results of regression analysis. Statistical analysis of differences between paired or unpaired means was carried out using $t$ test. Unless otherwise indicated, all data are presented as the mean \pm SD with the number of determinations given in parentheses.

\section{Results}

Correlation between cholesterol synthesis rates and plasma $M V A$ concentrations. We have previously compared plasma MVA concentrations with the rate of whole body cholesterol synthesis, which was measured by sterol balance, in three patients before and during treatment with cholestyramine (6). Table I summarizes these previous results together with new data that was obtained in studies of two patients before and after portacaval anastomosis (20), in four patients who received various amounts of dietary cholesterol, and in three patients who had high rates of cholesterol synthesis due to obesity. Taken together, these interventions provided a tenfold range of whole body cholesterol synthesis rates for comparison with plasma MVA concentrations. As shown in Fig. 1, the plasma MVA concentration was linearly related to the rate of cholesterol synthesis over the entire range from 4.7 to $53.2 \mathrm{mg}$ / $\mathrm{kg} \cdot \mathrm{d}$. By linear regression analysis, we found this relationship to be significant (slope $=3.63$, intercept $=5.69, r=0.972, P$ $<0.001, n=18 ; r_{s}=0.933$ ).

Diurnal variation of plasma MVA levels. On the basis of the above observations, we conclude that changes measured in plasma MVA at 7-9 a.m. are related meaningfully to variations in the rate of whole body cholesterol synthesis. However, plasma MVA concentrations are not constant throughout the day, but vary over a two- to fourfold range through each 24-h period, with peak levels occurring between midnight and 4 a.m. $(6,7)$. In Fig. 2 we show the patterns of plasma MVA concentrations that were measured in patient HF when he was eating ad lib three times a day as an outpatient, or 5 feedings/d when he was on the metabolic ward. These data demonstrate that plasma MVA concentrations can vary diurnally in free-living adults, much as they do in patients who were studied on the metabolic ward (6).

In Table II we compare daily whole body cholesterol synthesis rates and the mean concentration of MVA measured throughout a 24-h period in patient HF and two other patients: the two sets of measurements appear to be closely related (slope $=3.5$, intercept $=16.6, r=0.992, P<0.001, n=5$; $\left.r_{s}=1.0\right)$. Thus, changes in whole body cholesterol synthesis are reflected by corresponding changes in plasma MVA which was measured two ways: either at a fixed time of day (7-9 a.m.: mean of four or more days) (Fig. 1), or as the mean of 24 samples taken hourly through a single day (Table II).

Isotopic MVA kinetics. On the supposition that the rate of MVA turnover might indicate the rate of whole body cholesterol synthesis, we measured the rate of MVA input to the plasma compartment by the method of constant isotope infusion. Patient GDR was adapted to the metabolic ward (five liquid formula feedings per day for $24 \mathrm{~d}$ ) and received tracer 3R[5$\left.{ }^{3} \mathrm{H}\right] \mathrm{MVA}$ in an experiment that was carried out in the late morning to avoid the period of most rapid diurnal variation in plasma MVA concentrations. During the 7-h test period, his plasma MVA concentrations fluctuated between 19 and $22 \mathrm{nM}$, and an isotopic steady state was achieved between the fourth and sixth hours of constant intravenous infusion (Fig. 3). Under these conditions, the rate of net MVA input was $5.78 \mu \mathrm{mol} / \mathrm{d}$. The rate of whole body cholesterol synthesis, which was determined by simultaneous sterol balance measurements, was $5.7 \mathrm{mg} / \mathrm{kg} \cdot \mathrm{d}$ or $1.26 \mathrm{mmol} / \mathrm{d}$. The rate of MVA synthesis that was needed to account for daily cholesterol production, assuming a stoichiometry of $6 \mathrm{~mol}$ of MVA per mole of cholesterol, was $7.56 \mathrm{mmol} / \mathrm{d}$. Therefore, the daily flux of MVA through the plasma compartment was exceedingly small relative to its flux into cholesterol synthesis: $5.78 \mu \mathrm{mol}$ vs. $7.56 \mathrm{mmol}$ or $1 \mathrm{part} / 1,300$.

Effect of mevinolin on MVA. As a further test of the proposal that plasma MVA levels reflect cholesterol synthesis rates in the whole body, we carried out a series of studies with a specific inhibitor of HMG-CoA reductase, the drug mevinolin (11). The immediate effect of a single dose of placebo or 100 $\mathrm{mg}$ of mevinolin on successive occasions 1 wk apart to a group of five healthy volunteers is shown in Fig. 4. Although plasma MVA levels fell in the 6-h period after administration of either placebo or mevinolin, the changes observed after placebo $(31 \%$ 
Table I. Patient Data

\begin{tabular}{|c|c|c|c|c|c|c|}
\hline \multicolumn{3}{|l|}{ Physical } & \multicolumn{2}{|l|}{ Clinical } & \multicolumn{2}{|c|}{ Laboratory } \\
\hline Patient* & Age & Weight & Diagnosis & $\begin{array}{l}\text { Treatment } \\
\text { group }\end{array}$ & $\begin{array}{l}\text { Sterol } \\
\text { balanceł }\end{array}$ & $\begin{array}{l}\text { Plasma } \\
\text { MVA£ }\end{array}$ \\
\hline
\end{tabular}

Cholestyramine treatment

\begin{tabular}{|c|c|c|c|c|c|c|}
\hline PW & 35 & 91 & $\begin{array}{l}\text { HTG-CHO } \\
\text { Obese }\end{array}$ & $\begin{array}{l}\text { Control } \\
8 \mathrm{~g} \text { b.i.d. }\end{array}$ & $\begin{array}{l}20.7 \pm 1.2 \\
47.0 \pm 12.0\end{array}$ & $\begin{array}{c}72 \pm 7 \\
192 \pm 21\end{array}$ \\
\hline $\mathrm{CH}$ & 53 & 73 & $\mathrm{HC}$ & $\begin{array}{l}\text { Control } \\
8 \mathrm{~g} \text { b.i.d. }\end{array}$ & $\begin{array}{l}16.2 \pm 2.0 \\
27.6 \pm 7.0\end{array}$ & $\begin{array}{l}63 \pm 6 \\
92 \pm 41\end{array}$ \\
\hline $\mathbf{R M}$ & 57 & 67 & HTG-CHO & $\begin{array}{l}\text { Control } \\
8 \mathrm{~g} \text { b.i.d. }\end{array}$ & $\begin{array}{l}17.6 \pm 0.9 \\
53.2 \pm 8.0\end{array}$ & $\begin{array}{r}67 \pm 13 \\
203 \pm 28\end{array}$ \\
\hline \multicolumn{7}{|c|}{ Portacaval shunt } \\
\hline $\mathbf{A M}$ & $\begin{array}{l}4.5 \\
7.0\end{array}$ & $\begin{array}{l}19 \\
25\end{array}$ & HmFH & $\begin{array}{l}\text { Control } \\
\text { Post }(2 \text { y) }\end{array}$ & $\begin{array}{r}14.6 \pm 3.4 \\
4.7 \pm 0.6\end{array}$ & $\begin{array}{l}68 \pm 9 \\
26 \pm 2\end{array}$ \\
\hline PC & $\begin{array}{l}51 \\
53\end{array}$ & $\begin{array}{l}72 \\
68\end{array}$ & HtFH & $\begin{array}{l}\text { Control } \\
\text { Post(1.5 y) }\end{array}$ & $\begin{array}{l}8.9 \pm 1.3 \\
5.2 \pm 1.5\end{array}$ & $\begin{array}{l}63 \pm 7 \\
27 \pm 1\end{array}$ \\
\hline \multicolumn{7}{|c|}{ Various dietary cholesterol intakes" } \\
\hline EF & 45 & 82 & HC & 80 & $10.6 \pm 1.2$ & $48 \pm 10$ \\
\hline GDR & 52 & 66 & HTG-CHO & 490 & $7.4 \pm 0.9$ & $28 \pm 2$ \\
\hline HF & 68 & 84 & HTG & $\begin{array}{l}207 \\
972\end{array}$ & $\begin{array}{l}9.0 \pm 0.3 \\
6.7 \pm 1.0\end{array}$ & $\begin{array}{l}40 \pm 15 \\
28 \pm 6\end{array}$ \\
\hline $\mathbf{R} \mathbf{M}^{\prime}$ & 57 & 67 & HTG-CHO & 981 & $18.9 \pm 3.8$ & $60 \pm 7$ \\
\hline \multicolumn{7}{|c|}{ Obesity** } \\
\hline EK & 66 & 120 & $\mathbf{N}$ & 164 & $28.5 \pm 6.4$ & $88 \pm 12$ \\
\hline MP & 43 & 122 & $\mathbf{N}$ & 224 & $19.0 \pm 5.4$ & $91 \pm 23$ \\
\hline SN & 36 & 80 & $\mathbf{N}$ & 155 & $12.9 \pm 7.5$ & $39 \pm 5$ \\
\hline
\end{tabular}

HTG-CHO, hypertriglyceridemia induced by dietary carbohydrates; HC, hypercholesterolemia; N, normolipidemia without known atherosclerotic vascular disease; $\mathrm{HmFH}$, homozygous familial hypercholesterolemia; $\mathrm{HtFH}$, heterozygous familial hypercholesterolemia, as distinguished by LDL-receptor studies (20). Data taken from published studies: sterol balance and MVA data of patients PW, CH, and RM (6); and sterol balance but not MVA data of patients AM and PC (20). * All patients were males except for patients MP and SN. ₹ Sterol balance data are expressed as the mean \pm SD of measurements carried out on four or more 4-d fecal collections. § Plasma MVA data represent the mean \pm SD of determinations made on four or more samples between 7 and 9 a.m. after overnight fasts. "Intake of dietary cholesterol (mg/d). T The high levels of sterol balance in this patient, despite a high dietary cholesterol intake, may be due to his abnormally low absorption of dietary cholesterol $\left(8.96 \%\right.$ absorption $\times 981 \mathrm{mg}$ intake per day $=88 \mathrm{mg}$ absorbed per day). ${ }^{* *}$ Percentage of ideal body weight was calculated from the Metropolitan Life Table, Statistical Bulletin 40, November-December, 1959, Metropolitan Life Insurance Co., New York City.

decrease) were relatively small and were well within the range of diurnal variations that were described above. In contrast, plasma MVA concentrations were reduced $78 \%$ by the administration of mevinolin and were significantly different from those observed after placebo at all times between 2 and $6 \mathrm{~h}$. There was evidence of recovery of plasma MVA to pretreatment levels at $24 \mathrm{~h}$, but additional studies are needed to characterize the kinetics of this process. 


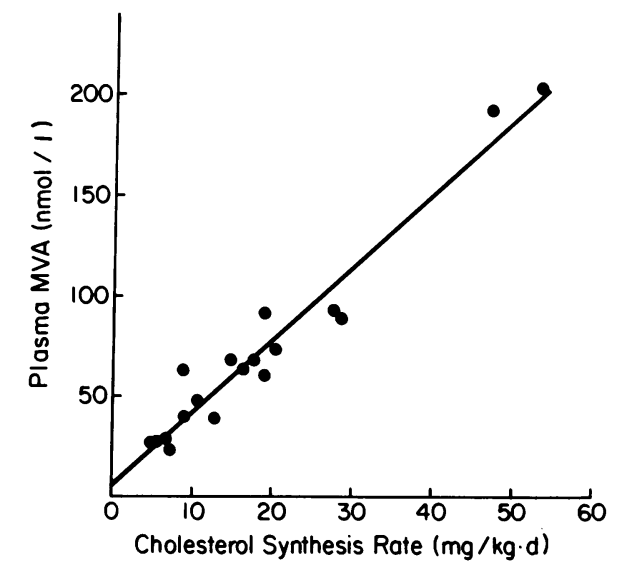

Figure 1. Rate of whole body cholesterol synthesis measured by sterol balance in 18 studies of 11 patients, compared with the concentration of MVA measured in plasma that was obtained from patients between 7 and 9 a.m. after an overnight fast. The solid line represents the best linear fit that was obtained by regression analysis: slope $=3.63 \pm 0.2(\mathrm{nmol} / \mathrm{l}) /(\mathrm{mg} / \mathrm{kg} \cdot \mathrm{d}) ;$ intercept $=5.69 \pm 4.8 \mathrm{nM}$; correlation coefficient $\left(r=0.972 ; P<0.001 ; r_{3}=0.933\right)$. A sterol synthesis rate of $1 \mathrm{mg} / \mathrm{kg} \cdot \mathrm{d}$ is equivalent to synthesis of $2.59 \mu \mathrm{mol} /$ $\mathbf{k g} \cdot \mathbf{d}$ of cholesterol.

Fig. 5 demonstrates the time courses of changes in concentrations of mevinolin in plasma after oral doses of 100 or 200 mg in relation to plasma MVA levels; all changes were dose related. The respective maximum and minimum levels occurred between 4 and $6 \mathrm{~h}$ after administration of drug; in addition, the 200-mg dose produced higher mevinolin concentrations and lower MVA levels through the entire 24-h period of study.

The rapid lowering of plasma MVA concentrations that was observed in these experiments, which was caused by a drug that was known to inhibit HMG-reductase, is strong evidence that short-term changes in the rate of MVA synthesis in tissues are quickly reflected by corresponding variations in the rate of MVA efflux and plasma concentration. These experiments also suggested to us that single doses of mevinolin had a short-lived effect on MVA production.

As a further study of the effect of mevinolin administration on MVA metabolism, we measured 24-h urinary MVA output as a function of drug dosage, since Kopito and Brunengraber (21) had shown that MVA output into urine is directly related to its concentration in plasma. As seen in Fig. 6, at the higher dose levels of 25 and $50 \mathrm{mg}$ twice a day, urinary MVA output was decreased relative to that seen in patients who received placebo. These differences were statistically significant, even when the data obtained in three patients who had MVA outputs $>2$ SD from their respective group means were not excluded (Table III). Regression analysis demonstrated a significant negative correlation between mevinolin dose and 24h urinary MVA output in all cases: $n=38$, slope $=-0.821$, intercept $=3.64, r=0.409$, and $P<0.02$; and in the cases

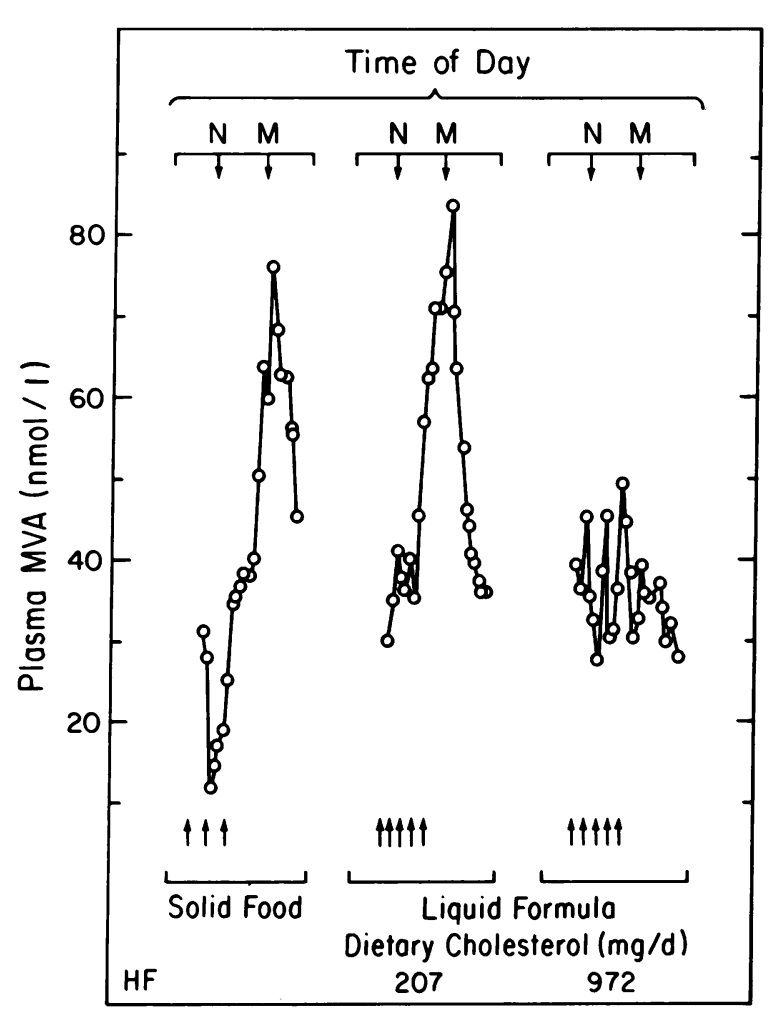

Figure 2. Hourly variations in plasma MVA concentration over three 24-h periods in patient HF. Left, when eating three daily meals at times shown by arrows as an outpatient; middle, after 4 wk on the metabolic ward with five equal-portion feedings that provided a total daily cholesterol intake of $207 \mathrm{mg} / \mathrm{d}$, which were consumed at 8 and $10,1,5$, and 7 p.m., and right, after a second 4-wk period on the metabolic ward during which the five equal-portion feedings provided $972 \mathrm{mg} / \mathrm{d}$ of dietary cholesterol.

remaining after the outliers' were excluded: $n=35$, slope $=-0.345$, intercept $=2.00, r=0.430$, and $P<0.02$.

Mevinolin, MVA, and cholesterol lowering. To examine the relationship between the effect of mevinolin on MVA and its ability to lower plasma low density lipoprotein (LDL) levels, we plotted the change in MVA and LDL for groups that received placebo, or $6.25,12.5,25$, and $50 \mathrm{mg}$ of mevinolin twice daily (Fig. 7). Increasing doses of mevinolin produced steadily decreasing 24-h urinary outputs of MVA, but changes in plasma LDL cholesterol levels reached a plateau at doses of $6.25 \mathrm{mg}$ twice daily. Therefore, in healthy subjects without FH a near-maximal LDL lowering by mevinolin was achieved with doses that only moderately reduced MVA synthesis.

\section{Discussion}

MVA and cholesterol synthesis. There is now a large body of evidence which demonstrates that MVA is formed in a reaction between HMG-CoA reductase and NADPH that is catalyzed 
Table II. Comparison of the Daily Rate of Whole Body Cholesterol Synthesis with the Mean of 24 Hourly Plasma Levels of MVA

\begin{tabular}{lll}
\hline Patient & Sterol balance* & $\begin{array}{l}\text { Mean daily plasmaf } \\
\text { MVA }\end{array}$ \\
\hline & $m g / k g \cdot d$ & $n m o l / l$ \\
EK & $28.5 \pm 6.4$ & $115 \pm 33$ \\
& $(4)$ & \\
EF & $10.6 \pm 1.0$ & $56 \pm 13$ \\
& $(6)$ & \\
HF§ & $9.0 \pm 0.3$ & $50 \pm 16$ \\
& $(4)$ & \\
HF§ & $6.7 \pm 10$ & $36 \pm 6$ \\
& $(5)$ &
\end{tabular}

* All values are expressed as mean $\pm \mathrm{SD}$; the number of 4-d fecal collections that were analyzed is given in parentheses.

$\ddagger$ Daily plasma MVA concentrations are the mean \pm SD of 24 hourly measurements; those for patients EK and EF were calculated from diurnal rhythms that were previously described by Parker et al. (6). $\S$ Patient HF was studied during periods of low and high dietary cholesterol intake (see Table I).

by HMG-CoA reductase, and that this reaction, under most physiological conditions, determines the rate of cholesterol synthesis. On the basis of previous work (6), we have proposed that variations in the plasma concentration of MVA reflect changes in the rate of whole body cholesterol synthesis; that this occurs because a small fraction of the MVA that is produced inside cells for cholesterol synthesis escapes from tissues to the plasma compartment; and, therefore, that mea-

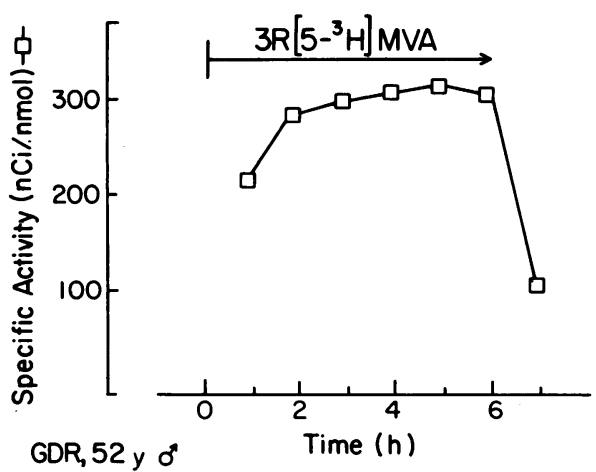

Figure 3. Plasma MVA specific activity ( $\mathrm{nCi} / \mathrm{nM}$ ) during administration of $3 \mathrm{R}-\left[5-{ }^{3} \mathrm{H}\right] \mathrm{MVA}(2,000 \mathrm{nCi} / \mathrm{nmol}$, sp act) by constant intravenous infusion $(1.27 \mu \mathrm{Ci} / \mathrm{min})$ after a priming dose of $40 \mu \mathrm{Ci}$ in patient GDR. Samples were taken from the contralateral antecubital vein at the times shown and assayed for MVA mass and radioactivity.

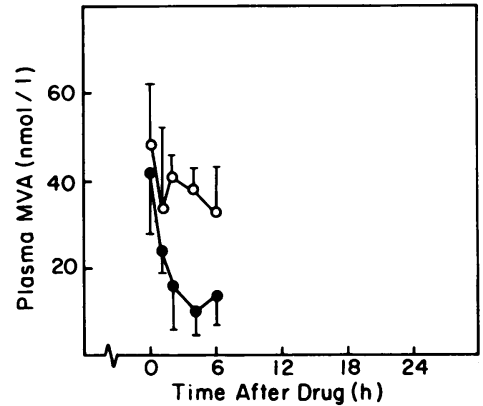

Figure 4. Variations in plasma MVA (mean \pm SD) in a group of five healthy male outpatients who received a single dose of placebo (o) or $100 \mathrm{mg}$ of mevinolin (๑). Either placebo or drug were administered (double blind) to fasting patients at 8 a.m., followed 2 and $4 \mathrm{~h}$ later by breakfast and a light lunch, respectively. Patients were discharged overnight and returned to the clinic the next morning for the 24-h blood sample. $1 \mathrm{wk}$ later, the contrasting medication was administered. Differences between placebo and drug periods are signifcantly different $(P<0.05)$ at 2,4 , and $6 \mathrm{~h}$.

surement of plasma MVA concentrations or 24-h urinary MVA output might prove useful as a means of estimating the rate of cholesterol synthesis in man. These hypotheses are supported by the data reported here: (a) plasma MVA concentrations measured at a fixed time of day (7-9 a.m.) are directly related to cholesterol synthesis rates over a tenfold range; $(b)$ only a small fraction $(\sim 1$ part/1,000) of the MVA made each day for cholesterol production escapes from the cells to plasma; and $(c)$ administration of mevinolin, a specific inhibitor of HMG-CoA reductase, causes a rapid and profound lowering

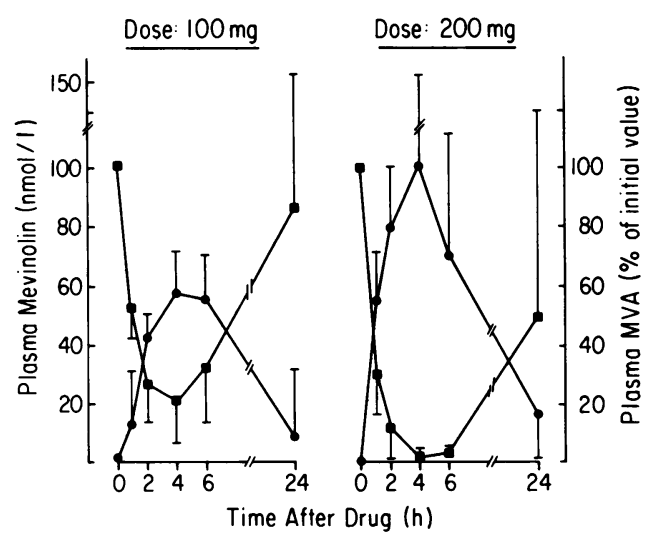

Figure 5. Effect of oral mevinolin on plasma concentration of mevinolin ( $\bullet$ ) and MVA ( $(\mathbf{)})$ in five subjects who received a 100-mg dose (left) and in another three subjects who received a 200-mg dose (right). Drug and blood samples were administered and collected as described in Fig. 4 and the patients who received 100-mg dose of mevinolin are the same. Data are plotted as means with the standard deviation shown by error bars. Plasma MVA concentrations are shown as percentage of the pretreatment values in order to combine data obtained from patients with different initial plasma MVA concentrations: the means and ranges were 40 (33-61) nM and 63 (37106) $\mathrm{nM}$ for the groups that received 100 or $200 \mathrm{mg}$, respectively. 


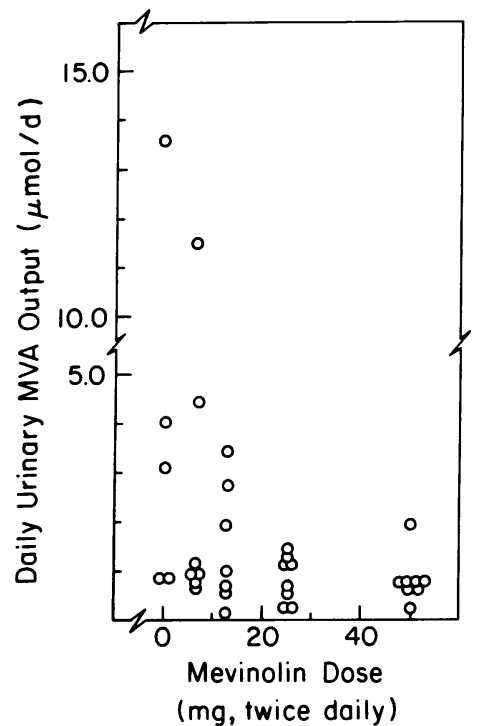

of plasma MVA concentrations and 24-h urinary MVA excretions.

The regression line that is shown in Fig. 1 predicts that plasma MVA concentrations decrease at very low rates of cholesterol synthesis, but not to zero. Extrapolation to the intercept at zero cholesterol synthesis by linear regression analysis predicts plasma MVA concentrations of $5.7 \pm 4.8 \mathrm{nM}$ $(P<0.2)$, when all data in Table $I$ are included, and 16.2 \pm 5.6 $\mathrm{nM}(P<0.2)$ when the highest cholesterol synthesis rates are excluded (cholestyramine periods of patients PW and RM). Although we cannot accept or reject the hypothesis of a nonzero intercept on the basis of available data, continuing MVA production in the absence of net sterol synthesis could have been anticipated from studies in cultured cells that have

Table III. Long-Term Effect of Mevinolin on 24-H Urinary MVA Output

\begin{tabular}{lll}
\hline \multirow{3}{*}{$\begin{array}{c}\text { Mevinolin dose } \\
\text { (mg twice daily) }\end{array}$} & \multicolumn{2}{l}{ Urinary MVA output } \\
\cline { 2 - 3 } & All subjects & Outliers excluded* \\
\hline & $\mu m o l / d$ & $\mu m o l / d$ \\
Placebo & $4.48 \pm 5.3 \quad(n=6)$ & $2.19 \pm 1.6 \quad(n=5)$ \\
6.25 & $3.12 \pm 3.9 \quad(n=7)$ & $1.56 \pm 1.6 \quad(n=6)$ \\
12.5 & $1.42 \pm 1.16 \quad(n=8)$ & $1.42 \pm 1.16 \quad(n=8)$ \\
25.0 & $0.83 \pm 0.46 \ddagger(n=8)$ & $0.83 \pm 0.46 \ddagger(n=8)$ \\
50.0 & $0.84 \pm 0.48 \ddagger(n=8)$ & $0.69 \pm 0.12 \S(n=7)$
\end{tabular}

* Individual values that differed from the group mean by two or more standard deviations were excluded (see $n$, which equals number of patients for each group).

₹ Significantly different from the placebo group $(P<0.05 ; t$ test). $\S$ Significantly different from the placebo group $(P<0.025 ; t$ test $)$.

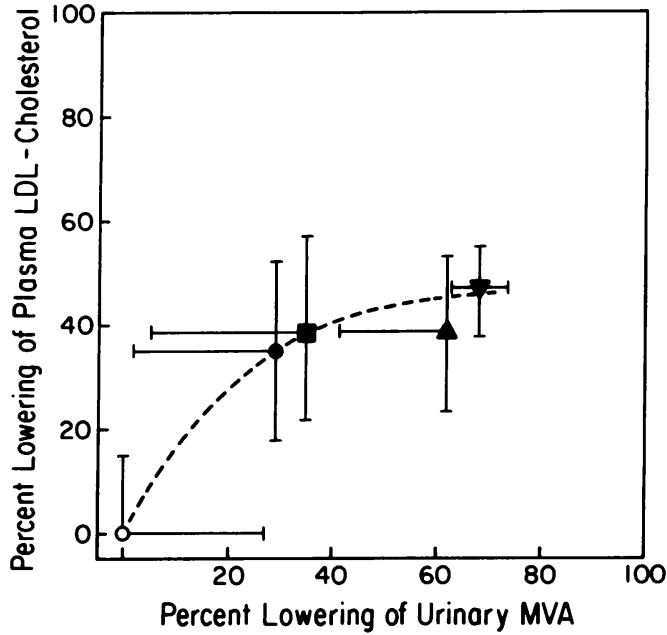

Figure 7. Comparison of the reductions in MVA and LDL-cholesterol caused by mevinolin at various dose levels. Changes in urinary MVA output are calculated as percentage $( \pm S D)$ of output in the placebo group from the right-hand column of Table III, and changes in plasma LDL-cholesterol are presented as percentage $( \pm S D)$ of the pretreatment level according to Fig. 2 of Tobert et al. (12). The data shown correspond to placebo (o), and to mevinolin doses of $6.25(\bullet)$, $12.5(\square), 25(\Delta)$, and $50(\nabla) \mathrm{mg}$ b.i.d., respectively.

shown that MVA synthesis cannot be fully suppressed by cholesterol alone: MVA synthesis is regulated to assure a continuing supply of ubiquinone, isopentenyl adenine, dolichols, and other nonsterol products that are derived from MVA (22). Other explanations of the nonzero intercept must also be considered. Net daily secretion by skin of squalene and sterols that are derived from MVA, which is estimated to be $<5 \%$ of total body cholesterol production (23), is not routinely included in measurements of whole body cholesterol production that are carried out by the sterol balance method. However, if MVA that was made in the metabolically active cells of the skin had access to the plasma compartment, even though the sterol made by the skin does not fully exchange with plasma cholesterol (24), this might explain the nonzero intercept.

Plasma MVA rhythm. The current studies of diurnal changes in plasma MVA concentrations extend the previous observations of Kopito and Brunengraber (7) and ourselves (6) by demonstrating that such rhythms can occur in freeliving adults. Moreover, the study of patient HF, in which we observed a nearly complete suppression of the midnight rise in plasma MVA concentrations (Fig. 2), a lowering of the 24 $\mathrm{h}$ mean MVA level, and a corresponding reduction in cholesterol production (Table II), provides additional support for the hypothesis that these short-term changes in plasma MVA are related to diurnal changes in cholesterol synthesis.

Comparison of the patterns of plasma MVA rhythms that were observed in detailed studies of the rat provides further insight on the origin of the rhythm in man. In the rat, 
cholesterol synthesis rates are high compared with man (60$100 \mathrm{mg} / \mathrm{kg} \cdot \mathrm{d}$ vs. $10-11 \mathrm{mg} / \mathrm{kg} \cdot \mathrm{d})$, and most of it $(60-80 \%)$ occurs in extrahepatic extraintestinal sites that are not subject to diurnal regulation $(25,26)$; diurnal changes in plasma MVA concentrations are found in the rat, but do not rise to statistical significance above high base-line levels (7). Finally, in the rat, dietary cholesterol suppresses hepatic sterol synthesis, but has little effect on synthesis elsewhere, whereas fasting suppresses synthesis in all tissues (25-27, and citations therein). In contrast, we have found low base-line plasma MVA concentrations in man (30-60 vs. $150-300 \mathrm{nM}$ in the rat); a pronounced diurnal rhythm that rises two- to fourfold above baseline plasma MVA concentrations; and suppression by dietary cholesterol of the postmidnight rise in plasma MVA to base line, but not to zero, levels. These findings in man, as contrasted to those in the rat, are consistent with: the lower rate of whole body cholesterol synthesis in man, constant but low rates of synthesis in peripheral tissues (especially in adults), and diurnal variations in sterol synthesis in liver and other tissues that are sensitive to feedback regulation by dietary cholesterol.

Plasma MVA flux. Others have shown that turnover of labeled MVA that is injected into the plasma compartment is characterized by a half-life time of $<40 \min (5,7,28)$ : rapid enough to reflect short-term changes in MVA input, such as those seen in the 24-h rhythm studies. In the studies reported here, MVA specific activity was measured in order to calculate daily MVA flux through the plasma compartment. This study demonstrated that only $\sim 1$ part/1,000 of the total MVA production enters plasma. Thus, labeled MVA that was injected into the plasma compartment does not trace the turnover of tissue MVA. Further, kinetic modeling and isotope kinetic studies are needed to learn why this is so.

In interpreting this finding we have considered two hypotheses: all of the MVA made by a small mass of tissue enters plasma, but none from other tissues, or else a small amount of MVA escapes from all tissues. We favor the latter because MVA and MVA lactone dissolve easily in polar and nonpolar solvents, and readily cross cell membranes (29). Moreover, passive transport across the cell membrane would provide a general mechanism for the escape of MVA in similar proportions from all cells.

Mevinolin, $M V A$, and $L D L$ lowering. As judged by its effect on plasma MVA concentrations, mevinolin is an effective inhibitor of MVA production in healthy men. Single doses of mevinolin caused a rapid lowering of the MVA that was maintained for at least $6 \mathrm{~h}$. Plasma MVA returned to pretreatment levels $24 \mathrm{~h}$ after dosing, with evidence of individual variation that was reflected in the relative large standard deviations of the 24-h MVA data in Figs. 4 and 5. Taken together with the finding that diurnal rhythms of plasma MVA occur in free-living adults, these observations suggest that the time of day may be an important determinant of the extent to which net daily MVA synthesis and plasma LDL concentrations are lowered by mevinolin administration.
The effect of compactin, another inhibitor of HMG-CoA reductase, on the diurnal rhythm of cholesterol synthesis, has been characterized in the rat. Single oral doses of compactin inhibited cholesterol synthesis in all tissues for the first few hours after administration. This was followed by a period of super-normal synthesis, such that net daily cholesterol synthesis and plasma lipid levels were unchanged $(30,31)$ or only slightly lowered (32).

In man, the time-averaged effect of one or more doses of mevinolin can be estimated from changes in 24-h urinary MVA outputs. Low doses of mevinolin (6.25-12.5 mg b.i.d.) appeared to cause relatively minor reductions in MVA synthesis, yet achieved near maximal LDL lowering. High doses (50 mg b.i.d.) markedly reduced MVA synthesis, but added little to the LDL-lowering effect. These observations lead us to conclude that relatively small reductions in MVA (or cholesterol) production can trigger significant changes in lipoprotein metabolism in normal subjects. Even at the highest dose, mevinolin did not suppress MVA synthesis to levels that would be expected to limit polyisoprenoid biosynthesis (22).

Sterol synthesis: measurement by sterol balance vs. estimation from MVA measurements. We believe that the results that are reported here demonstrate that measurements of plasma or urinary MVA can provide additional information regarding cholesterol synthesis rates that are not available through any other technique: this was made most evident in the studies of diurnal MVA rhythms and the short-term changes in plasma MVA after administration of mevinolin.

The extent to which MVA measurements can substitute for sterol balance measurements remains unclear. The sterol balance method provides a direct quantitative measurement of average daily cholesterol production, as well as information regarding sterol and bile acid metabolism. Quantitation of cholesterol synthesis from MVA measurements is indirect: plasma MVA concentrations that are measured between 7 and 9 a.m. after an overnight fast, 24-h mean plasma MVA concentration, or 24-h urinary MVA outputs are translated into estimates of cholesterol synthesis by use of a calibration curve such as that shown in Fig. 1. Application of this procedure is based on several assumptions, two of the most important being that within narrow limits, from one subject to the next, the same fraction of total cellular MVA production must escape into plasma, and that renal function and hence MVA clearance and excretion is normal. These assumptions must be shown to be true for each new clinical state before MVA data can be used to infer absolute rates of cholesterol synthesis.

Certainly, MVA measurements can be used to document two- to threefold changes in cholesterol synthesis, such as seen after PCA or administration of cholestyramine: smaller changes in cholesterol synthesis are detectable under controlled circumstances, such as those attained on the metabolic ward. In outpatient studies, where random variations in the timing of the diurnal rhythm are likely to increase the coefficient of variation in measurements of plasma MVA, useful information 
can be obtained from measurements of 24-h MVA outputs (see Table III and Fig. 5). Where the collection of 24-h urine pools is difficult or impossible, plasma MVA concentrations that are measured in control and experimental periods may be compared and expressed as relative change. This experimental design allows the patient to serve as his own control, and thereby reduces error from sources such as differences in MVA transport or catabolism between individuals. We anticipate that MVA transport or catabolism will differ substantially from normal in some disease states, and may obscure the relationship between MVA and cholesterol synthesis that was demonstrated here. We therefore recommend that plasma or urinary MVA data be compared with sterol balance in each new patient group or disease state before conclusions regarding synthesis are drawn from MVA data alone.

\section{Acknowledgments}

We thank Dr. Banvir S. Chaudhary, of The Rockefeller University Computing Services, J. A. Bolognese and M. Stat. of Merck Sharp \& Dohme Research Laboratories, for their advice and assistance in carrying out the statistical analyses, and Dr. Ralph Dell of Columbia University for his help in modeling MVA turnover kinetics. We acknowledge the excellent technical assistance of Lie Ping Li, Anna Adamo, Eleanor Mathusek, Lyudmila Malikin, Florence Chu, and the services of The Rockefeller University Hospital nursing and dietary staffs.

This work was supported in part by U. S. Public Health Service grants HL 24190, from the National Heart Lung and Blood Institute, FR 00102, from the General Clinical Research Centers Branch of the Division of Research Resources, and CA 295902, from the National Cancer Institute; this work was also funded by grants from the Herman Goldman Foundation, the Jean and Louis Dreyfus Foundation, and the National Dairy Council.

\section{References}

1. Grundy, S. M., E. H. Ahrens, Jr., and J. Davignon. 1969. The interaction of cholesterol absorption and cholesterol synthesis in man. J. Lipid Res. 10:304-315.

2. Grundy, S. M., E. H. Ahrens, Jr., and G. Salen. 1971. Interruption of the enterohepatic circulation of bile acids in man: comparative effects of cholestyramine and ileal exclusion on cholesterol metabolism. J. Lab. Clin. Med. 78:94-121.

3. Dietschy, J. M., and J. D. Wilson. 1970. Regulation of cholesterol metabolism. N. Engl. J. Med. 282:1128-1138, 1179-1183, 1241-1249.

4. Hagenfeldt, L., and K. Hellstrom. 1972. Blood concentration and turnover of circulating mevalonate in the rat. Life Sci. Part 2 Physiol. Pharmacol. 11:669-676.

5. Popják, G., G. Boehm, T. S. Parker, J. Edmond, P. Edwards, and A. M. Fogelman. 1979. Determination of mevalonate in blood plasma in man and rat. Mevalonate 'tolerance' tests in man. J. Lipid Res. 20:716-728.

6. Parker, T. S., D. J. McNamara, C. Brown, O. Garrigan, R. Kolb, H. Batwin, and E. H. Ahrens, Jr. 1982. Mevalonic acid in human plasma: relationship of concentration and circadian rhythm to cholesterol synthesis rates in man. Proc. Natl. Acad. Sci. USA. 79:30373041.
7. Kopito, R. R., B. Weinstock, L. E. Freed, D. M. Murray, and H. Brunengraber. 1982. Metabolism of plasma mevalonate in rats and humans. J. Lipid Res. 23:577-583.

8. Wiley, M. H., M. M. Howton, and M. D. Siperstein. 1977. The quantitative role of the kidneys in the in vivo metabolism of mevalonate. J. Biol. Chem. 252:548-554.

9. Brunengraber, H., S. B. Weinstock, D. L. Story, and R. R. Kopito. 1981. Urinary clearance and metabolism of mevalonate by isolated perfused rat kidney. J. Lipid Res. 22:916-920.

10. Schwartz, C. C., M. Berman, Z. R. Vlahcevic, D. Halloran, H. Gregory, and L. Swell. 1978. Multicompartmental analysis of cholesterol metabolism in man. J. Clin. Invest. 61:408-423.

11. Alberts, A. W., J. Chen, G. Kuron, V. Hunt, J. Huff, C Hoffman, J. Rothrock, M. Lopez, H. Joshua, E. Harris, A. Patchett, R. Monaghan, S. Currie, E. Stapley, G. Albers-Schonberg, O. Hensens, J. Hirshfield, K. Hoogsteen, J. Liesch, and J. Springer. 1980. Mevinolin: a highly potent competitive inhibitor of hydroxymethylglutaryl-coenzyme A reductase and a cholesterol-lowering agent. Proc. Natl. Acad. Sci. USA. 77:3957-3961.

12. Tobert, J. A., G. D. Bell, J. Birtwell, I. James, W. R. Kukovetz, J. S. Pryor, A. Buntinx, I. B. Holmes, Y.-S. Chao, and J. A. Bolognese. 1982. Cholesterol-lowering effect of mevinolin, an inhibitor of 3hydroxy-3-methylglutaryl-coenzyme A reductase, in healthy volunteers. J. Clin. Invest. 69:913-919.

13. Ahrens, E. H., Jr., V. P. Dole, and D. H. Blankenhorn. 1954. The use of orally-fed liquid formulas in metabolic studies. Am. J. Clin. Nutr. 2:336-342.

14. Miettinen, T. A., E. H. Ahrens, Jr., and S. M. Grundy. 1965. Quantitative isolation and gas chromatographic analysis of total dietary and fecal neutral steroids. J. Lipid Res. 6:411-424.

15. Grundy, S. M., E. H. Ahrens, Jr., and T. A. Miettinen. 1965. Quantitative isolation and gas-liquid chromatographic analysis of total fecal bile acids. J. Lipid Res. 6:397-410.

16. Davignon, J., W. J. Simmonds, and E. H. Ahrens, Jr. 1968. Usefulness of chromic oxide as an internal standard for balance studies in formula-fed patients and for assessment of colonic function. J. Clin. Invest. 47:127-138.

17. Grundy, S. M., E. H. Ahrens, Jr., and G. Salen. 1968. Dietary $\beta$-sitosterol as an internal standard to correct for cholesterol losses in sterol balance studies. J. Lipid Res. 9:374-387.

18. Popják, G. 1969. Enzymes of sterol biosynthesis in liver and intermediates of sterol biosynthesis. Methods Enzymol. 15:393-454.

19. Shapiro, D. J., J. L. Nordstrom, J. J. Mitschelin, V. W. Rodwell, and R. T. Schimke. 1974. Microassay for 3-hydroxy-3methylglutaryl CoA reductase in rat liver and in L-cell fibroblasts. Biochim. Biophys. Acta. 370:369-377.

20. McNamara, D. J., E. H. Ahrens, Jr., R. Kolb, T. S. Parker, P. Samuel, C. D. Brown, N. E. Davidson, and R. McVie. 1983. Treatment of familial hypercholesterolemia by portacaval anastomosis: effect on cholesterol metabolism and pool sizes. Proc. Natl. Acad. Sci. USA. 80:564-568.

21. Kopito, R. R., and H. Brunengraber. 1980. (R)-mevalonate excretion in human and rat urines. Proc. Natl. Acad. Sci. USA. 77:5738-5740.

22. Brown, M. S., and J. L. Goldstein. 1980. Multivalent feedback regulation of HMG-CoA reductase, a control mechanism coordinating isoprenoid synthesis and cell growth. J. Lipid Res. 21:505-517.

23. Nikkari, T., P. H. Schreibman, and E. H. Ahrens, Jr. 1974. In vivo studies of sterol and squalene secretion by human skin. J. Lipid Res. 15:563-573. 
24. Nikkari, T., P. H. Schreibman, and E. H. Ahrens, Jr. 1975. Isotope kinetics of human skin cholesterol secretion. J. Exp. Med. 141:620-634.

25. Jeske, D. J., and J. M. Dietschy. 1980. Regulation of rates of cholesterol synthesis in vivo in the liver and carcass of the rat measured using ${ }^{3} \mathrm{H}$ water. J. Lipid Res. 21:364-376.

26. Feingold, K., M. H. Wiley, G. Macrae, S. Lear, A. H. Moser, G. Zsigmond, and M. D. Siperstein. 1983. De novo sterologenesis in the intact rat. Metab. Clin. Exp. 32:75-81.

27. Edwards, P. A., H. Muroya, and G. Gould. 1972. In vivo demonstration of the circadian rhythm of cholesterol biosynthesis in the liver and intestine of the rat. J. Lipid Res. 13:396-401.

28. Hellstrom, K. H., M. D. Siperstein, L. A. Bricker, and L. J. Luby. 1973. Studies of the in vivo metabolism of mevalonic acid in the normal rat. J. Clin. Invest. 52:1303-1313.

29. Edwards, P. A., J. Edmond, A. M. Fogelman, and G. Popják.
1977. Preferential uptake and utilization of mevalonolactone over mevalonate for sterol biosynthesis in isolated rat hepatocytes. Biochim. Biophys. Acta. 488:493-501.

30. Fears, R., D. H. Richards, and H. Ferres. 1980. The effect of compactin, a potent inhibitor of 3-hydroxy-3-methylglutaryl coenzymeA reductase activity and serum cholesterol levels in rats and chicks. Atherosclerosis. 35:439-449.

31. Bensch, W. R., T. S. Ingebritsen, and E. R. Diller. 1978. Lack of correlation between the rate of cholesterol biosynthesis and the activity of 3-hydroxy-3-methylglutaryl coenzyme $A$ reductase in rats and in fibroblasts treated with ML-236B. Biochem. Biophys. Res. Commun. 82:247-254.

32. Endo, A., Y. Tsujita, M. Kuroda, and K. Yansawa. 1979. Effects of ML-236B on cholesterol metabolism in mice and rats: lack of hypercholesterolemic activity in normal animals. Biochim. Biophys. Acta. 575:266-276. 